\title{
Wavelet-Based Compressed Sensing for SAR Tomography of Forested Areas
}

\author{
Esteban Aguilera, Matteo Nannini, and Andreas Reigber, Senior Member, IEEE
}

\begin{abstract}
Synthetic aperture radar (SAR) tomography is a 3-D imaging modality that is commonly tackled by spectral estimation techniques. Thus, the backscattered power along the cross-range direction can be readily obtained by computing the Fourier spectrum of a stack of multibaseline measurements. In addition, recent work has addressed the tomographic inversion under the framework of compressed sensing, thereby recovering sparse cross-range profiles from a reduced set of measurements. This paper differs from previous publications, in that it focuses on sparse expansions in the wavelet domain while working with the second-order statistics of the corresponding multibaseline measurements. In this regard, we elaborate on the conditions under which this perspective is applicable to forested areas and discuss the possibility of optimizing the acquisition geometry. Finally, we compare this approach with traditional nonparametric ones and validate it by using fully polarimetric L-band data acquired by the Experimental SAR (E-SAR) sensor of the German Aerospace Center (DLR).
\end{abstract}

Index Terms-Compressed sensing (CS), forest structure, synthetic aperture radar (SAR) tomography, wavelets.

\section{INTRODUCTION}

$\mathbf{S}$ YNTHETIC aperture radar (SAR) tomography allows for effective retrieval of cross-range scattering profiles from measurements obtained through repeat-pass acquisitions. Due to the high-penetration capabilities of radiation at long wavelengths, such as those within the L- or P-band, the complete spatial distribution of volumetric scatterers can be satisfactorily resolved. A case in point is the 3-D imaging of vegetated areas, which has proven to be of great value for the estimation of forest structure along with its underlying ground topography [1]-[7].

A common approach is to employ parallel tracks, thus rendering the tomographic inversion a direction-of-arrival problem. As a result, the estimation of the cross-range power distribution can be effectively tackled by spectral estimation techniques. However, the achievable resolution of conventional spectral estimators is highly dependent on the extension of the tomographic aperture (see Fig. 1). Moreover, the sampling rate dictated by the Nyquist frequency imposes an additional requirement, namely, dense regular sampling.

Manuscript received January 12, 2012; revised July 11, 2012 and October 8, 2012; accepted November 21, 2012.

The authors are with the Microwaves and Radar Institute (HR), German Aerospace Center (DLR), 82234 Wessling, Germany (e-mail: Esteban. Aguilera@dlr.de; Matteo.Nannini@dlr.de; andreas.reigber@dlr.de).

Color versions of one or more of the figures in this paper are available online at http://ieeexplore.ieee.org.

Digital Object Identifier 10.1109/TGRS.2012.2231081

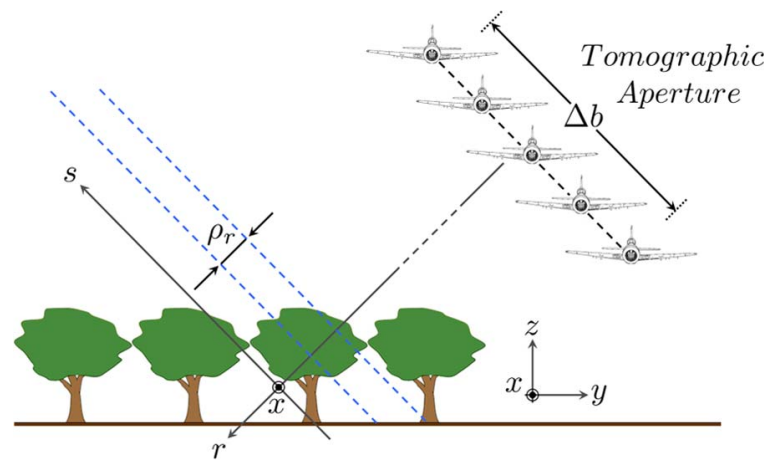

Fig. 1. Tomographic sensing operation using parallel passes (not to scale). Every pixel of each SAR image is a projection of the reflectivity along $s$ (crossrange). The range resolution is indicated by $\rho_{r}$, whereas the extension of the tomographic aperture is indicated by $\Delta b$.

Subsequent to the first demonstration of SAR tomography [1], [8], [9], several extensions and alternatives have been put forward in order to attain low sidelobe and ambiguity levels with a reduced number of irregular passes. The use of adaptive spectral estimators was introduced in [10] and [11] and further developed in [12] and [13] (see also [6]). In addition, subspace-based spectral estimators, such as the multiple signal classification algorithm, have been recently employed [6], [13]-[16]. In [17], the authors formulated the tomographic inversion under the framework of linear inverse problems, thus exploiting the truncated singular-value decomposition. Also, a maximum a posteriori estimator was developed in [18]. Other publications have addressed irregular geometries by means of interpolation techniques (see, for example, [7] and [19]). Alternatively, an extension of SAR interferometry from a parametric perspective was proposed in [20]. In a nutshell, this last work employs covariance matching estimation techniques in order to estimate the effective scattering center of different scattering mechanisms, along with their backscattered power. Moreover, the author in [21] introduced the concept of polarization coherence tomography. Basically, the method exploits the variation of the interferometric coherence with polarization to estimate the ground topography and height of the vegetation layer. Then, it uses these parameters to represent a cross-range profile as a Fourier-Legendre series. Finally, sparsity-driven inversion techniques were introduced in [22]-[25]. In essence, the authors applied the relatively new compressed sensing (CS) theory to achieve superresolution imaging. Nevertheless, the signals of interest were sparse in the space domain, a situation that is rarely true when it comes to vegetated areas.

In this paper, we formulate the retrieval of the cross-range power distribution of forested areas under the framework of CS. 
In light of previous work in the context of SAR [26]-[29] and other fields (see, for example, [30] and [31]), we exploit sparse representations in the wavelet domain. Furthermore, we work with second-order statistics, since, under certain assumptions on the covariance matrix of the unknown tomographic signal, we can interpret the entries of the multibaseline covariance matrix as measurements of the cross-range power distribution. As a consequence, this approach also allows us to use a small number of irregular baselines to optimize the tomographic acquisition.

The remainder of this paper is organized as follows. Section II formulates the tomographic sensing problem from a covariance matrix perspective. Section III provides a short introduction to CS theory and wavelets. In addition, in Section IV, we regard the tomographic problem as an instance of CS and propose a sparsifying basis which thrives on the simplicity of the cross-range power distribution. In Section V, we draw a comparison with traditional nonparametric spectral estimators and present results obtained using fully polarimetric L-band data acquired by one of the airborne sensors of the German Aerospace Center (DLR), namely, Experimental SAR (E-SAR). Lastly, Section VI concludes this paper.

\section{PRoblem Formulation}

We are interested in reconstructing the 3-D power distribution $p(x, r, s)$ of a complex reflectivity function $g(x, r, s)$, where $x, r$, and $s$ are the azimuth, range, and cross-range coordinates, respectively (see Fig. 1). At a specific azimuthrange position, the corresponding discretized signals along $s$, with $1 \leq s \leq n$, will be denoted with the column vectors $p \in$ $\mathbb{R}_{\geq 0}^{n}$ and $g \in \mathbb{C}^{n}$.

For the sake of simplicity, we will, for now, neglect any source of decorrelation [32], although it will be incorporated in the tomographic reconstruction (see Section IV-A). Under this hypothesis, the tomographic acquisition using $m$ parallel tracks can be modeled as

$$
b=\Phi g
$$

where $b \in \mathbb{C}^{m}$ is a stack of pixels taken from $m$ focused and coregistered SAR images. The matrix $\Phi \in \mathbb{C}^{m \times n}$ is the socalled steering matrix which accounts for the phase rotations due to the distance traveled by the microwave pulses from the sensor to the targets distributed along $s$ and back to the sensor [15]. In addition, the covariance matrix $C \in \mathbb{C}^{m \times m}$, corresponding to partial scatterers, can be written out as follows:

$$
C=E\left\{b b^{*}\right\}=\Phi \operatorname{diag}(p) \Phi^{*}
$$

where $E\{\cdot\}$ is the expectation operator, $(\cdot)^{*}$ denotes the conjugate transpose, and $\operatorname{diag}(p) \in \mathbb{R}_{\geq 0}^{n \times n}$ is a matrix whose main diagonal equals $p$ and contains zeros in its off-diagonal elements [2], [20]. Thus, we can attempt to recover $p$ based on the entries of $C$.

Alternatively, we can form $C_{h h}, C_{v v}$, and $C_{h v}$, where $h h$, $v v$, and $h v$ indicate polarization diversity, and add them to form $C_{\text {span }}$. In effect, since

$$
C_{h h}+C_{v v}+C_{h v}=\Phi \operatorname{diag}\left(p_{h h}+p_{v v}+p_{h v}\right) \Phi^{*}
$$

TABLE I

LIST OF SYMBOLS-SECTION II

\begin{tabular}{lr}
\hline$x$ & $\begin{array}{r}\text { azimuth coordinate } \\
\text { range coordinate }\end{array}$ \\
$s$ & cross-range coordinate \\
$m$ & number of parallel passes \\
$n$ & length of cross-range signal \\
$b \in \mathbb{C}^{m}$ & multibaseline measurements \\
$g \in \mathbb{C}^{n}$ & cross-range complex reflectivity \\
$p \in \mathbb{R}_{\geq 0}^{n}$ & cross-range power distribution \\
$\Phi \in \mathbb{C}^{m \times n}$ & steering matrix \\
$C \in \mathbb{C}^{m \times m}$ & multibaseline covariance matrix \\
\hline
\end{tabular}

and therefore

$$
C_{\text {span }}=\Phi \operatorname{diag}\left(p_{\text {span }}\right) \Phi^{*}
$$

it follows that the estimation of $p_{\text {span }}$ from $C_{\text {span }}$ will simply represent the polarimetric span. See Table I for a quick reference to this section.

\section{CS AND WAVELETS}

\section{A. $C S$}

CS is a sampling paradigm that allows us to capture a signal of interest at a rate significantly below the Nyquist one. It enables us to go beyond the Shannon limit by exploiting sparse representations [33]-[36]. In particular, a signal $f \in \mathbb{C}^{N}$ is said to be $K$-sparse in an orthonormal basis $\Psi \in \mathbb{C}^{N \times N}$ if its projection $\alpha=\Psi f \in \mathbb{C}^{N}$ has, at most, $K$ nonzero elements. In turn, we have $f=\Psi^{*} \alpha$. Thus, CS proposes measuring such a signal $f$ by collecting $M$ linear measurements of the form $b=A f+y$ or $b=A \Psi^{*} \alpha+y \in \mathbb{C}^{M}$, where $A \in \mathbb{C}^{M \times N}$ is a sensing matrix with $M$ much smaller than $N$ and $y \in \mathbb{C}^{M}$ is a perturbation term. Also, we define $\Theta=A \Psi^{*} \in \mathbb{C}^{M \times N}$, so that $b=\Theta \alpha+y$. In addition, the matrix $\Theta$ obeys the restricted isometry property (RIP) of order $K$ if there exists a constant $\delta_{K} \in(0,1)$ such that

$$
\left(1-\delta_{K}\right)\|\alpha\|_{2}^{2} \leq\|\Theta \alpha\|_{2}^{2} \leq\left(1+\delta_{K}\right)\|\alpha\|_{2}^{2}
$$

holds for all $K$-sparse signals $\alpha$. This property essentially requires that every set of, at most, $K$ columns approximately behaves like an orthonormal system [37], [38]. As developed in [39] and [40], if $\Theta$ satisfies the RIP of order $2 K$ with $\delta_{2 K}<\sqrt{2}-1$, then we can recover $\alpha$ from the measurements $b$ by $L_{1}$ norm minimization

$$
\min _{\widetilde{\alpha}}\|\widetilde{\alpha}\|_{1} \text { subject to }\|\Theta \widetilde{\alpha}-b\|_{2} \leq \varepsilon
$$

and the solution $\widetilde{\alpha}$ obeys

$$
\|\widetilde{\alpha}-\alpha\|_{2} \leq C_{0}\left\|\alpha-\alpha_{K}\right\|_{1} / \sqrt{K}+C_{1} \varepsilon
$$

for some constant $C_{0}$ and $C_{1}$, where $\alpha_{K}$ is the signal $\alpha$ with all but the largest $K$ components set to zero and $\varepsilon$ is an upper 
TABLE II

List of SyMBOLS-SECTION III-A

\begin{tabular}{lr}
\hline$f \in \mathbb{C}^{N}$ & unknown signal \\
$\Psi \in \mathbb{C}^{N \times N}$ & sparsifying basis for $f$ \\
$\alpha \in \mathbb{C}^{N}$ & $K$-sparse transform coefficients of $f$ \\
$A \in \mathbb{C}^{M \times N}$ & sensing matrix \\
$y \in \mathbb{C}^{M}$ & perturbation term \\
$\Theta \in \mathbb{C}^{M \times N}$ & shorthand for $A \Psi^{*}$ \\
$\delta_{K} \in(0,1)$ & restricted isometry constant \\
$\varepsilon$ & upper bound on perturbation level \\
$\Upsilon \in \mathbb{C}^{N \times N}$ & dictionary of sampling waveforms \\
$\mathcal{F} \in \mathbb{C}^{N \times N}$ & shorthand for $\Upsilon \Psi^{*}$ \\
$\mu(\mathcal{F}) \in[1, \sqrt{N}]$ & coherence of $\mathcal{F}$ \\
\hline
\end{tabular}

bound on the perturbation level. In other words, this means that the largest $K$ nonzero elements are recovered in their correct location and that the error is proportional to the rest of the nonzero elements and the perturbation level. Finally, we recover $\widetilde{f}$ by computing $\widetilde{f}=\Psi^{*} \widetilde{\alpha}$.

As a result of the previous discussion, we would like to design a matrix $A$ for a given $\Psi$ such that $\Theta=A \Psi^{*}$ satisfies the RIP with $\delta_{2 K}<\sqrt{2}-1$. To that end, let $\Upsilon \in \mathbb{C}^{N \times N}$ be an orthonormal matrix and $\mathcal{F}=\Upsilon \Psi^{*} \in \mathbb{C}^{N \times N}$. Also, let us define the coherence of $\mathcal{F}$ as

$$
\mu(\mathcal{F})=\sqrt{N} \max _{i, j}\left|\mathcal{F}_{i, j}\right| \in[1, \sqrt{N}]
$$

which basically measures the largest correlation between the rows of $\Upsilon$ and $\Psi$ [41]. Then, it can be shown [37], [42] that, if we construct $A$ by taking

$$
M=\mathcal{O}\left(\mu^{2}(\mathcal{F}) K \log ^{4} N\right)
$$

sensing waveforms (rows) of $\Upsilon$ uniformly at random and renormalize the columns so that they are unit normed, then $\delta_{2 K}<\sqrt{2}-1$ holds with large probability. Table II provides a quick reference to this section.

\section{B. Wavelet Systems}

An orthogonal wavelet system is generally regarded as a set of functions used for uniquely representing a signal. When formulated from a multiresolution perspective, these functions can be divided into two classes, namely, scaling and wavelet functions, that represent coarse and fine information, respectively. Thus, the discrete wavelet transform (DWT) of a signal $f(t)$ for a given scale $j_{0}$ computes the coefficients $c_{j_{0}}$ and $d_{j}$-at $k$ different shifts—as follows:

$$
\begin{aligned}
c_{j_{0}}(k) & =\int f(t) \varphi_{j_{0}, k}(t) d t \\
d_{j}(k) & =\int f(t) \psi_{j, k}(t) d t
\end{aligned}
$$

where $j \in\left[j_{0},+\infty\right), k \in(-\infty,+\infty)$, and $\varphi_{j_{0}, k}(t)$ and $\psi_{j, k}(t)$ are the scaling and wavelet functions, respectively. Then, $f(t)$
TABLE III

List OF SYMBOLS-SECTION III-B

\begin{tabular}{lr}
\hline$f(t)$ & signal of interest \\
$j_{0}$ & scale for scaling coefficients \\
$j \in\left[j_{0},+\infty\right)$ & scale for wavelet coefficients \\
$k \in(-\infty,+\infty)$ & integer shift \\
$\varphi_{j_{0}, k}(t)$ & scaling function \\
$\psi_{j, k}(t)$ & wavelet function \\
$c_{j_{0}}$ & scaling coefficients at scale $j_{0}$ \\
$d_{j}$ & wavelet coefficients at scale $j$ \\
$v$ & vanishing moments \\
\hline
\end{tabular}

TABLE IV

LIST OF SYMBOLS-SECTION IV-A

\begin{tabular}{lr}
\hline$m$ & $\begin{array}{r}\text { number of parallel passes } \\
\text { length of cross-range signal }\end{array}$ \\
$p \in \mathbb{R}_{\geq 0}^{n}$ & cross-range power distribution \\
$\Phi \in \mathbb{C}^{m \times n}$ & steering matrix \\
$\Phi_{j} \in \mathbb{C}^{n}$ & baseline sensing waveform \\
$\xi_{j, k} \in \mathbb{C}^{n}$ & cobaseline sensing waveform \\
$\widehat{C} \in \mathbb{C}^{m \times m}$ & multibaseline sample covariance matrix \\
$\Psi \in \mathbb{R}^{n \times n}$ & sparsifying basis \\
$\varepsilon$ & upper bound on model mismatch \\
$\lambda_{1}$ & data mismatch penalty parameter \\
$\lambda_{2}$ & total-variation penalty parameter \\
\hline
\end{tabular}

can be recovered from

$$
f(t)=\sum_{k} c_{j_{0}}(k) \varphi_{j_{0}, k}(t)+\sum_{k} \sum_{j=j_{0}}^{+\infty} d_{j}(k) \psi_{j, k}(t)
$$

[43]. In addition, we will say that a wavelet with $v$ vanishing moments is orthogonal to polynomials of degree $v-1$ [44]. Hence, if $f(t)$ exhibits such a polynomial behavior, all its wavelet coefficients $d_{j}(k)$ will be zero, which, in turn, implies that $f(t)$ will be fully captured by the scaling coefficients $c_{j_{0}}(k)$. As a result, if $f(t)$ has few isolated singularities and is very regular, we should choose a wavelet with many vanishing moments in order to achieve a sparse expansion. On the contrary, if the number of singularities increases and we do not want the chosen wavelets to overlap these singularities (and thus create high-amplitude coefficients), we need to decrease the size of their support. Unfortunately, this is achieved at the expense of reducing the number of vanishing moments. That being said, we encounter a tradeoff between the number of vanishing moments and the support size [44]. Table III summarizes the concepts of this section.

\section{WAVELET-BASED CS FOR SAR TOMOGRAPHY}

\section{A. SAR Tomography as an Instance of CS}

By considering (2) and (6) together, we can readily recast the power distribution estimation as an instance of CS. Once an 

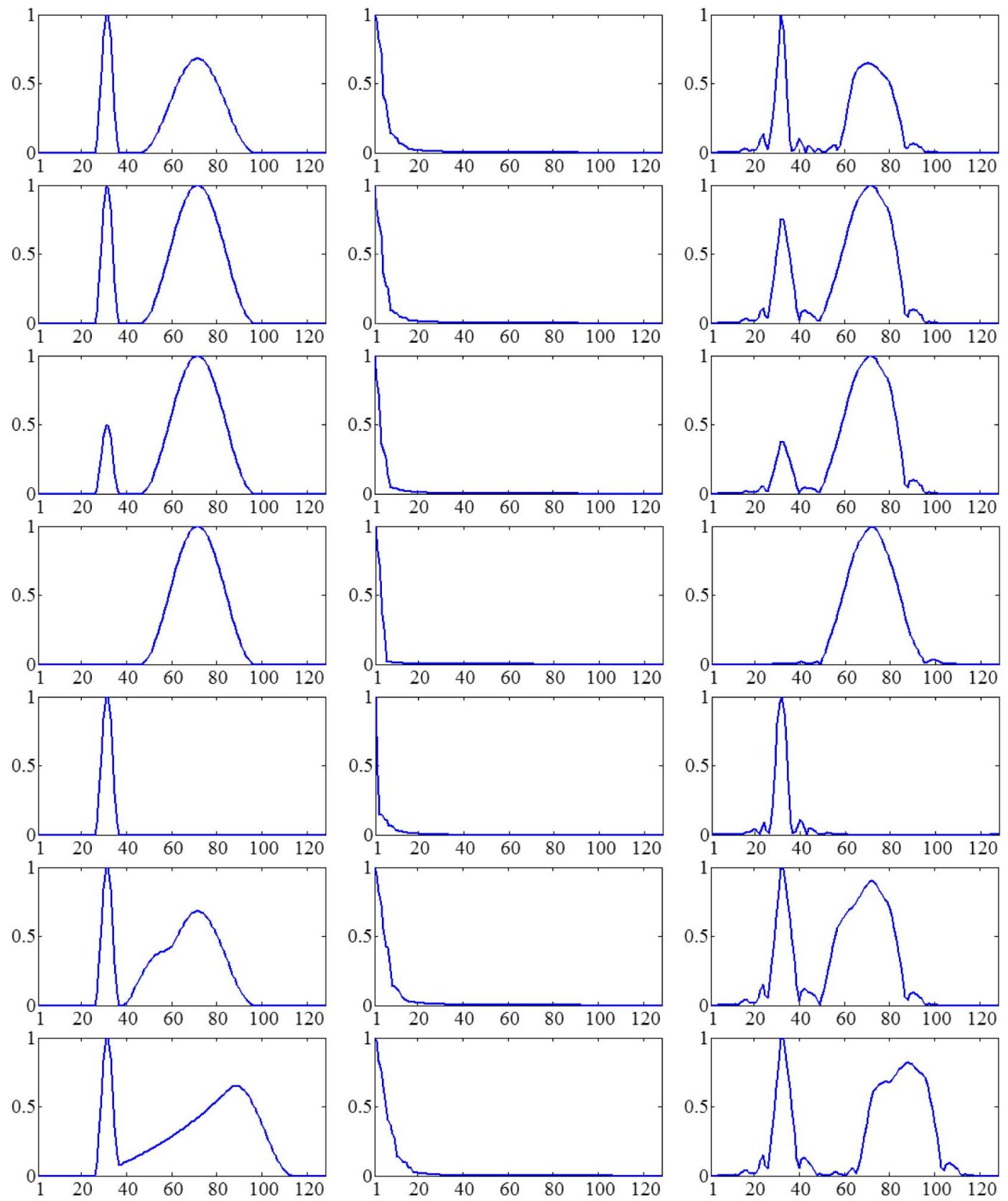

Fig. 2. Sparsity in the wavelet domain. (Left column) Power distribution (as a function of height with $n=128$ ) of typical cross-range profiles encountered over forested terrain. (Middle column) Sorted magnitudes of the transform coefficients using a Daubechies Symmlet wavelet with four vanishing moments and three levels of decomposition. (Right column) Magnitude of the inverse DWT after zeroing out all but the five largest coefficients. All plots have been normalized.

appropriate sparsifying basis $\Psi \in \mathbb{R}^{n \times n}$ has been chosen, we can formulate the reconstruction of $p$ as follows:

$$
\min _{\widetilde{p}}\|\Psi \widetilde{p}\|_{1} \text { subject to }\left\|\Phi \operatorname{diag}(\widetilde{p}) \Phi^{*}-\widehat{C}\right\|_{F} \leq \varepsilon
$$

where $\widehat{C}$ is the sample covariance matrix, $\|\cdot\|_{F}$ denotes the Frobenius norm, and $\varepsilon$ can be used to control the tradeoff between sparsity in $\Psi$ and model mismatch. In accordance with the definition of $p$, the optimization has to be carried out over the set of nonnegative real numbers.

It is worth mentioning that (13) implies a nonlinear reconstruction. Therefore, we might incur radiometric accuracy which, as denoted by (7), will be bounded by both $\varepsilon$ and the sparsity level. Thus, whereas the former is directly related to any source of decorrelation as well as to the number of looks that were used to compute the sample covariance matrix $\widehat{C}$, the latter translates into the number of effective unknowns, i.e., the coefficients that sparsely represent $p$.

In order to provide greater insight into this CS perspective, we can use (2) to express each entry of the covariance matrix $C$ as

$$
C_{j, k}=\left\langle p, \xi_{j, k}\right\rangle
$$

with

$$
\xi_{j, k}=\Phi_{j} \odot \operatorname{conj}\left(\Phi_{k}\right)
$$

where $1 \leq j, k \leq m,\langle\cdot, \cdot\rangle$ denotes the inner product, $\Phi_{j} \in \mathbb{C}^{n}$ represents the $j$ th row of $\Phi$, and $\odot$ indicates element-wise 
multiplication. With this in mind, the tomographic acquisition basically samples the unknown $p$ by computing inner products with $m^{2}$ sensing waveforms $\xi_{j, k} \in \mathbb{C}^{n}$. Also, as argued in [22], $\Phi$ behaves approximately like a partial Fourier matrix. Hence, the waveforms $\xi_{j, k}$ will be sinusoids as well. Accordingly, the coherence of $\mathcal{F}=\Upsilon \Psi^{*}$, as defined by (8), ought to be computed by letting $\Upsilon$ be a Fourier matrix. Furthermore, just as every signal $\Phi_{j}$ is directly related to a specific baseline $j$, we will consider every signal $\xi_{j, k}$ to be related to a specific cobaseline, which we define as the difference between the baselines $j$ and $k$. Consequently, when designing a baseline distribution, each resulting vector $\xi_{j, k}$, and not $\Phi_{j}$, should represent a row of $\Upsilon$ taken uniformly at random. As will be shown in Section V, in order to avoid redundancy, additional emphasis should be placed on the cobaselines. For details on this concept, known in the literature as minimum redundancy arrays, we refer the reader to [45] and [46].

Finally, we propose solving (13) in Lagrangian form, along with an additional total-variation (TV) norm regularization [47], which results in

$$
\min _{\widetilde{p}}\|\Psi \widetilde{p}\|_{1}+\lambda_{1}\left\|\Phi \operatorname{diag}(\widetilde{p}) \Phi^{*}-\widehat{C}\right\|_{F}^{2}+\lambda_{2}\|\widetilde{p}\|_{\mathrm{TV}}
$$

where

$$
\|p\|_{\mathrm{TV}}=\sum_{s=2}^{n}|p[s]-p[s-1]|
$$

and the parameters $\lambda_{1}$ and $\lambda_{2}$ control the tradeoff between sparsity in $\Psi$, model mismatch, and TV. Essentially, $\|\cdot\|_{\mathrm{TV}}$ enables us to introduce prior knowledge about the fact that the nonzero elements of $p$ tend to appear in groups (see Section IV-B), thereby exploiting the ordering of the features in $p$ [48]. See Table IV for a reference to this section.

\section{B. Choosing a Sparsifying Basis}

As a consequence of (9), the choice of an orthonormal basis $\Psi$ requires some special consideration. First, the signal $p$ must have a sparse expansion $\Psi p$. Second, we require that the coherence between the measurement basis $\Upsilon$ and the sparsity basis $\Psi$ be as small as possible. In this section, we will propose a sparsifying basis that is in line with these two requirements.

In the case of monostatic SAR acquisitions, cross-range profiles of forest canopy follow, in general, a very simple twocomponent structure. Specifically, one of these components accounts for ground backscattering, double-bounce scattering from ground-trunk interactions, and double-bounce contributions from ground-volume interactions, whereas the other accounts for volume backscattering. In fact, this model has been thoroughly discussed/validated at C-band [49], L-band [3], and P-band [4], [20]. Interestingly, the distribution of the effective scattering over forested terrain is quite regular, thereby giving rise to sparse representations in the wavelet domain. By way of illustration, the left column of the plots in Fig. 2 shows several commonly encountered profiles. In addition, the middle column displays the rapid decay of the sorted magnitudes of the corresponding transform coefficients, which were computed using

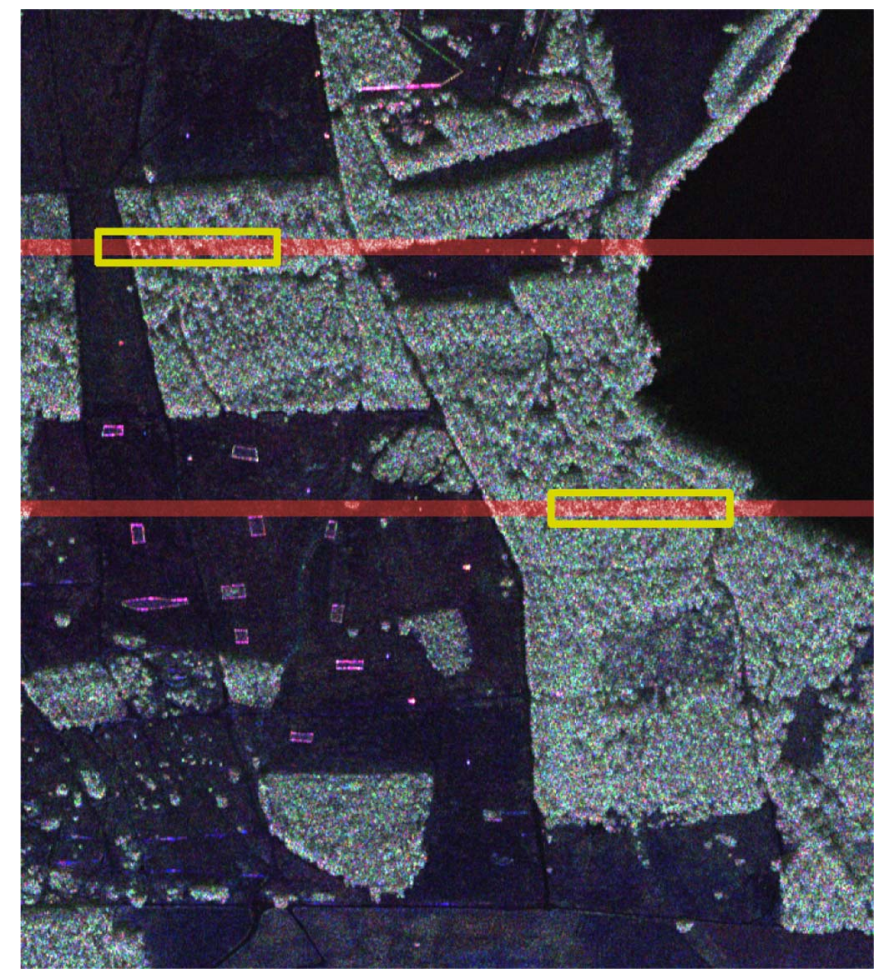

Fig. 3. Polarimetric SAR image of the test site near Dornstetten, Germany (red: $|h h-v v| / \sqrt{2}$; green: $\sqrt{2}|h v|$; and blue: $|h h+v v| / \sqrt{2}$ ). The targets of interest are located within the yellow rectangles along azimuth.

Daubechies Symmlet wavelets with four vanishing moments and three levels of decomposition. Finally, the right column presents the magnitude of the inverse DWT, after zeroing out all but the five largest transform coefficients. Clearly, the profiles are very well approximated. In this respect, we justify the choice of Symmlets by noting that, besides yielding good results in practice, they are optimal in the sense that they have minimum support for a given number of vanishing moments [43], [44].

Lastly, in order to generate the maximum number of small wavelet coefficients, we might be tempted to use a $\Psi$ that computes many levels of decomposition. However, more levels of decomposition lead to a higher coherence. For example, four levels result in $\mu=4.0$, three levels result in $\mu=2.8284$, and two levels result in $\mu=2.0$.

\section{EXPERIMENTAL RESUlts}

In order to demonstrate the advantages and the shortcomings of the outlined approach, we used simulated data as well as a stack of 21 focused and coregistered SAR images obtained by processing real fully polarimetric L-band data. These data were acquired by the E-SAR airborne sensor of DLR during a campaign near Dornstetten, Germany, in 2006. All flights were performed at approximately the same altitude with horizontal baselines of about $20 \mathrm{~m}$. Fig. 3 shows the amplitude image of this area. The center frequency used was $1.3 \mathrm{GHz}$, and the nominal altitude above ground was about $3200 \mathrm{~m}$. The resolutions were 0.66 and $2.07 \mathrm{~m}$ in azimuth and range, respectively. The near, middle, and far ranges corresponded to $3953.15,4527.09$, and $5102.52 \mathrm{~m}$, respectively [15]. We 


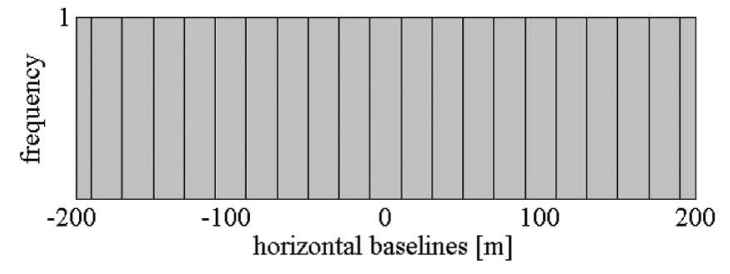

(a)

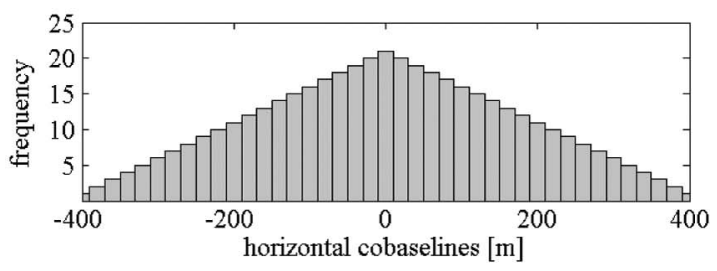

(b)

Fig. 4. Histogram of (a) 21 horizontal baselines employed in the first constellation and (b) resulting horizontal cobaselines.

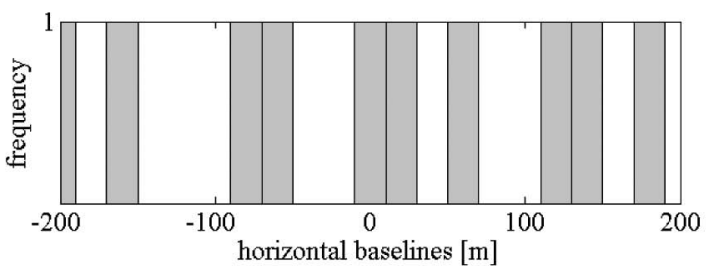

(a)

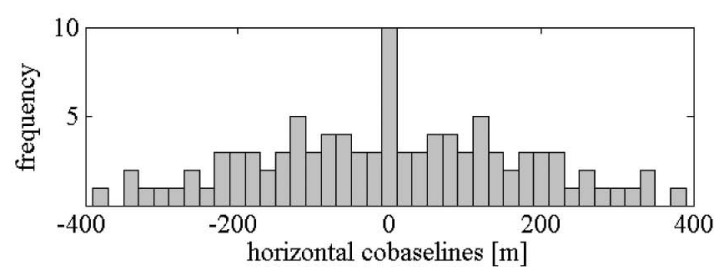

(b)

Fig. 5. Histogram of (a) ten horizontal baselines employed in the second constellation and (b) resulting horizontal cobaselines.

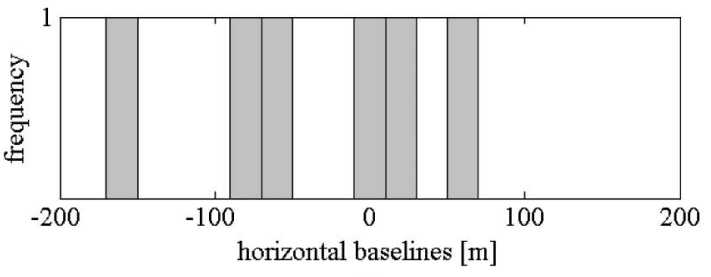

(a)

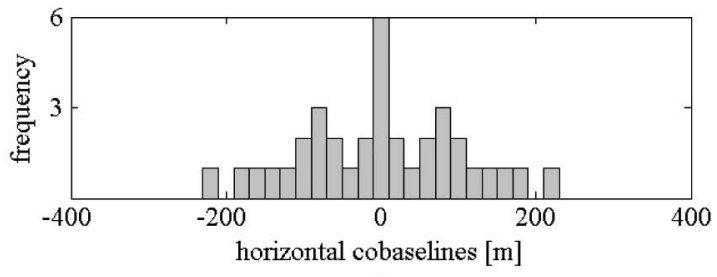

(b)

Fig. 6. Histogram of (a) six horizontal baselines employed in the third constellation and (b) resulting horizontal cobaselines.

considered three different constellations (which will be referred to throughout this section) employing the following: C1) all 21 passes; $C 2$ ) ten irregular passes; and C3) six irregular passes. Figs. 4-6 show the histograms of horizontal baselines and corresponding horizontal cobaselines. Note that Fig. 4(b) uncovers a high level of redundancy, unlike Figs. 5(b) and $6(b)$.

\section{A. Experiments With Simulated Data}

1) Description of the Experiments: We started by comparing two traditional nonparametric estimators, namely, Fourier beamforming and Capon's method, with the wavelet-based CS (WCS) technique letting $\lambda_{1}=\lambda_{2}=0.5$ [see (16)], and employing a Daubechies Symmlet wavelet with four vanishing moments and three levels of decomposition. To this end, we simulated a 300-look cross-range profile following a circular Gaussian distribution with zero mean and unit variance, so that (2) holds asymptotically. In addition, we generated multibaseline measurements considering the system parameters mentioned previously and the constellations $C 1-C 3$ at the near, middle, and far ranges. The decorrelation effects were introduced by means of Gaussian noise using a signal-to-noise ratio (SNR) of $10 \mathrm{~dB}$.

The tomographic inversion was carried out under different assumptions on the extent of the cross-range profile, i.e., the observation space. Fig. 7 shows the normalized profiles as a function of height obtained using 21 passes. First, we assumed an observation space of $80 \mathrm{~m}$ with $n=256$ at near, middle, and far ranges [see Fig. 7(a)-(c)]. Then, we restricted it to $40 \mathrm{~m}$ with $n=128$ [see Fig. 7(d)-(f)]. Similarly, we performed the reconstruction employing ten and six tracks (see Figs. 8 and 9). Alternatively, Fig. 10 shows an example of the impact of choosing an insufficient range of heights (i.e., $20 \mathrm{~m}$ with $n=128$ ), whereby a part of the backscatter is neglected.

2) Description of the Results: In light of these simulations, several observations can be made.

1) When using all the available passes (Fig. 7), WCS almost does not suffer from ambiguities. A further reduction of the observation space does not seem to provide any significant advantage.

2) When decreasing the number of passes to ten (Fig. 8), despite providing similar results to those obtained using 21 tracks, a further reduction of the range of heights does prove to be advantageous for WCS at the near range. The reconstruction is actually unsatisfactory if this is not taken into account [compare Fig. 8(a) and (d)].

3) A more limited number of tracks (Fig. 9) can lead to unsatisfactory results at the near range, regardless of our previous knowledge about the observation space [compare Fig. 9(a) and (d)].

4) An erroneous range of heights may introduce artifacts in the WCS reconstruction (see Fig. 10).

3) Discussion: It is known that, in SAR tomography, the order of the RIP might fall short of ideal, even when attaining 


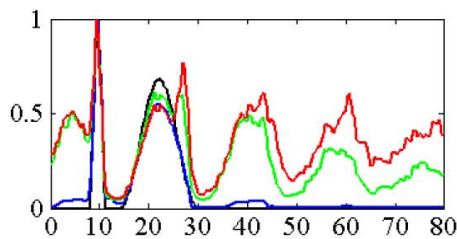

(a)

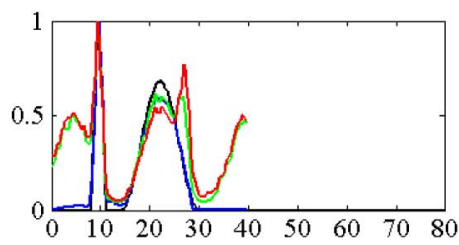

(d)

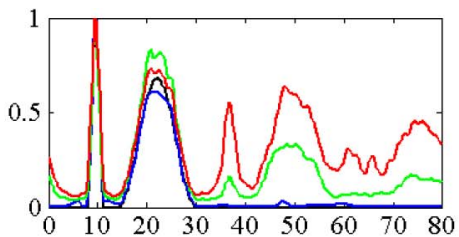

(b)

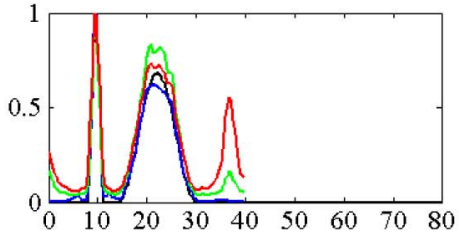

(e)

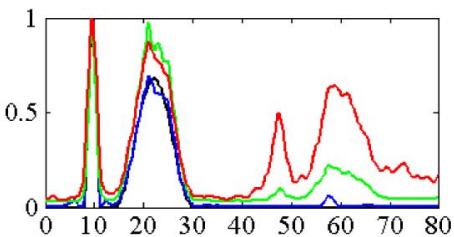

(c)

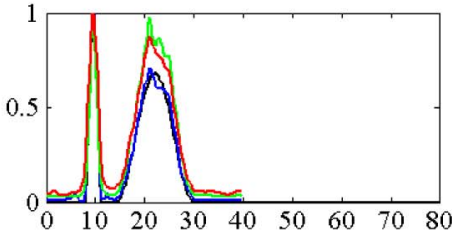

(f)

Fig. 7. Normalized cross-range profiles as a function of height (in meters) obtained using 21 passes, 300 looks, and SNR $=10 \mathrm{~dB}$. (Black) Simulated. (Blue) WCS. (Green) Capon's method. (Red) Fourier. (Top plots) An Observation space corresponding to a height range of $80 \mathrm{~m}$ has been considered at (a) near, (b) middle, and (c) far ranges. (Bottom plots) A Limited observation space corresponding to a height range of $40 \mathrm{~m}$ has been considered at (d) near, (e) middle, and (f) far ranges.

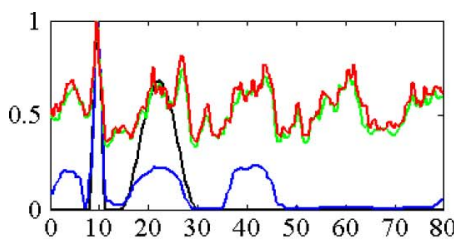

(a)

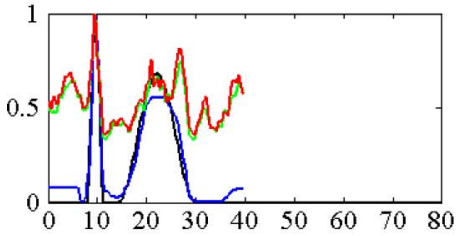

(d)

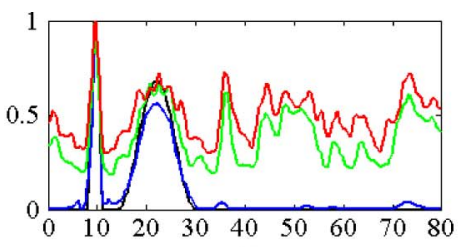

(b)

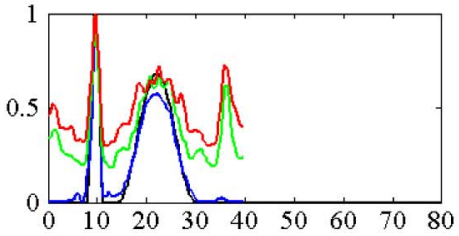

(e)

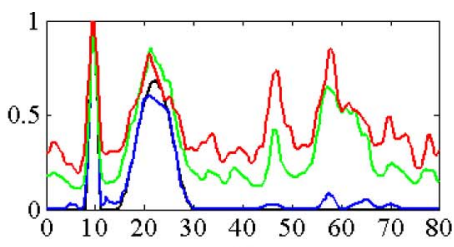

(c)

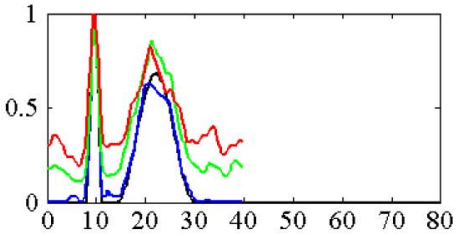

(f)

Fig. 8. Normalized cross-range profiles as a function of height (in meters) obtained using ten irregular passes, 300 looks, and SNR $=10 \mathrm{~dB}$. (Black) Simulated. (Blue) WCS. (Green) Capon's method. (Red) Fourier. (Top plots) An Observation space corresponding to a height range of $80 \mathrm{~m}$ has been considered at (a) near, (b) middle, and (c) far ranges. (Bottom plots) A Limited observation space corresponding to a height range of $40 \mathrm{~m}$ has been considered at (d) near, (e) middle, and (f) far ranges.

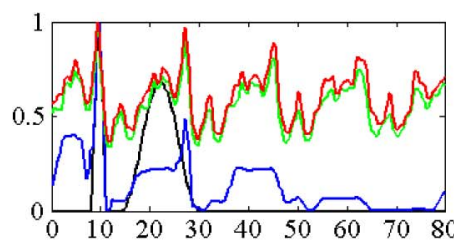

(a)

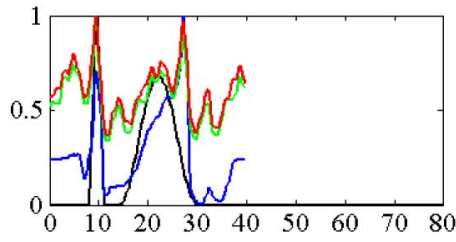

(d)

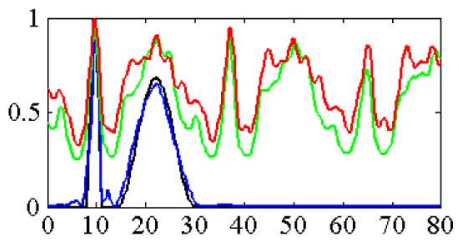

(b)

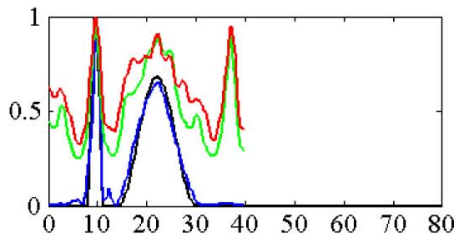

(e)

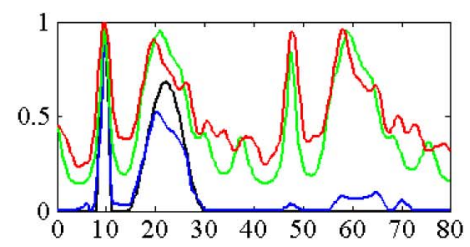

(c)

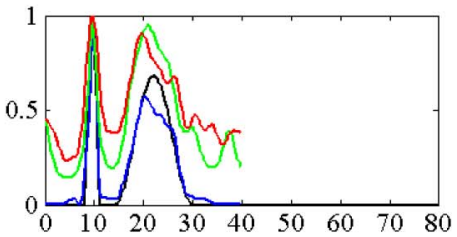

(f)

Fig. 9. Normalized cross-range profiles as a function of height (in meters) obtained using six irregular passes, 300 looks, and SNR $=10 \mathrm{~dB}$. (Black) Simulated. (Blue) WCS. (Green) Capon's method. (Red) Fourier. (Top plots) An Observation space corresponding to a height range of $80 \mathrm{~m}$ has been considered at (a) near, (b) middle, and (c) far ranges. (Bottom plots) A Limited observation space corresponding to a height range of $40 \mathrm{~m}$ has been considered at (d) near, (e) middle, and (f) far ranges.

optimal coherence between the measurement basis and the sparsity basis (see, for example, [24]). Nonetheless, the experiments outlined in this section (see observations $1-3$ ) indicate that this inherent limitation can be transcended (to a certain extent, depending on the number of available passes) by appropriately defining the range of heights. 


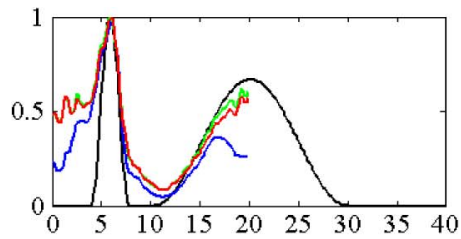

(a)

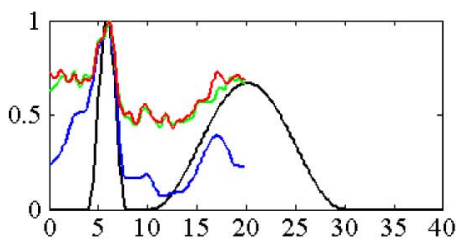

(d)

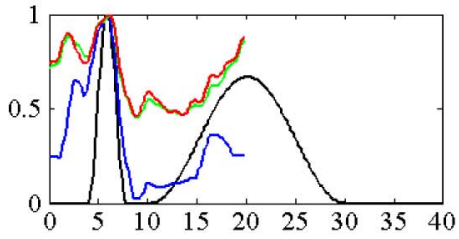

(g)

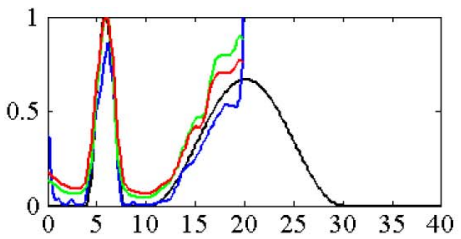

(b)

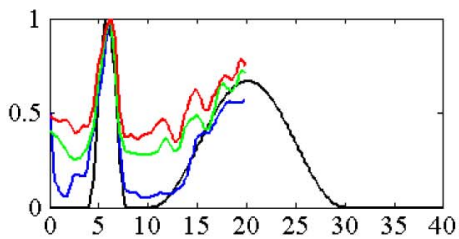

(e)

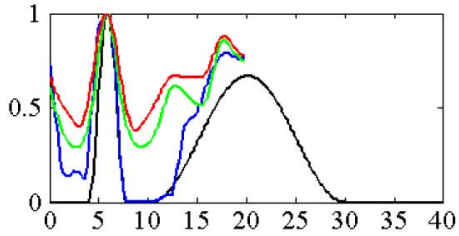

(h)

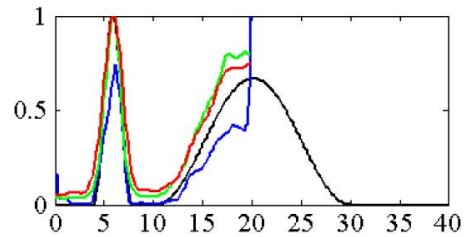

(c)

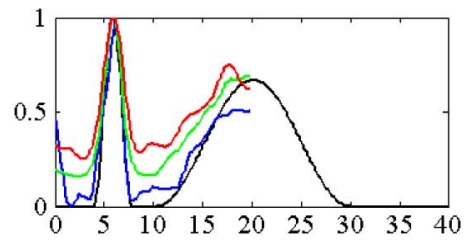

(f)

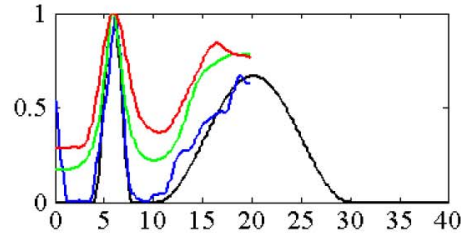

(i)

Fig. 10. Normalized cross-range profiles as a function of height (in meters) obtained using 300 looks, and SNR $=10 \mathrm{~dB}$. (Black) Simulated. (Blue) WCS. (Green) Capon's method. (Red) Fourier. The observation space corresponds to a height range of $20 \mathrm{~m}$, thus ignoring part of the cross-range backscatter. (Top plots) Twenty-one passes at (a) near, (b) middle, and (c) far ranges. (Middle plots) Ten irregular passes at (d) near, (e) middle, and (f) far ranges. (Bottom plots) Six irregular passes at $(\mathrm{g})$ near, $(\mathrm{h})$ middle, and (i) far ranges.

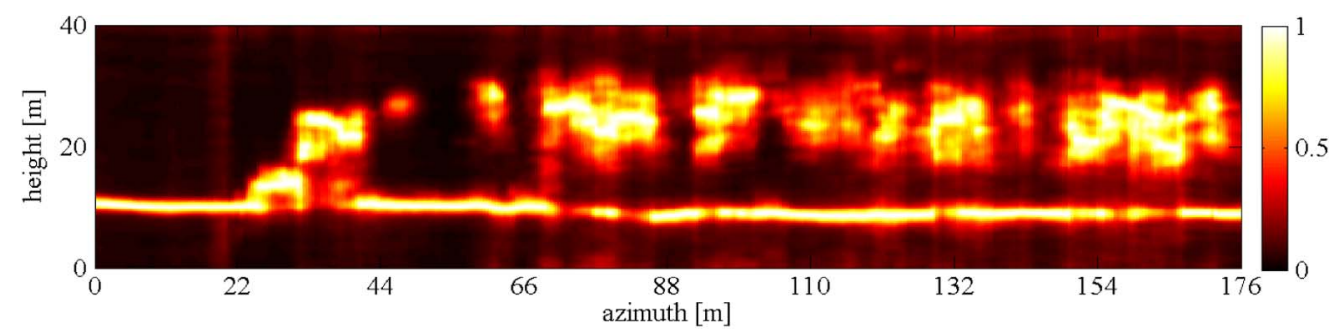

(a)

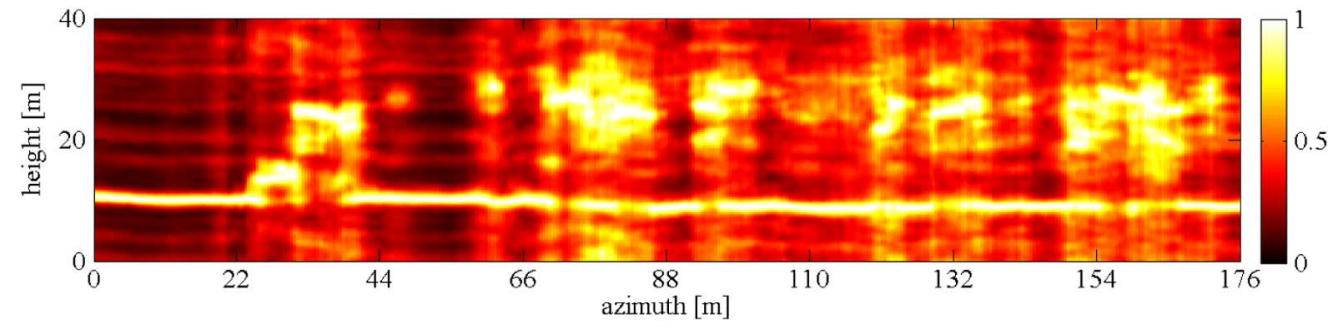

(b)

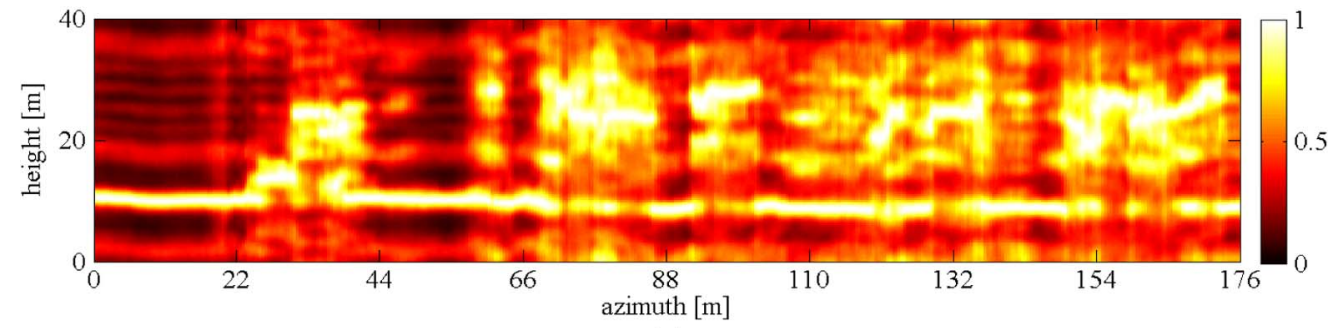

(c)

Fig. 11. Span of tomogram obtained by Fourier beamforming as a function of azimuth and height (176 m by $40 \mathrm{~m}$ ) using a nine-by-nine window with (a) 21 , (b) 10, and (c) 6 passes. Range distance: $4816.30 \mathrm{~m}$.

Of equal importance is the fact that, unlike Fourier beamforming and Capon's method, WCS retrieves the backscattered power simultaneously for all heights in the defined observa- tion space. While this is one of the reasons why ambiguities are countered, an erroneous range of heights impacts on the WCS reconstruction (see observation 4). Nevertheless, this is 


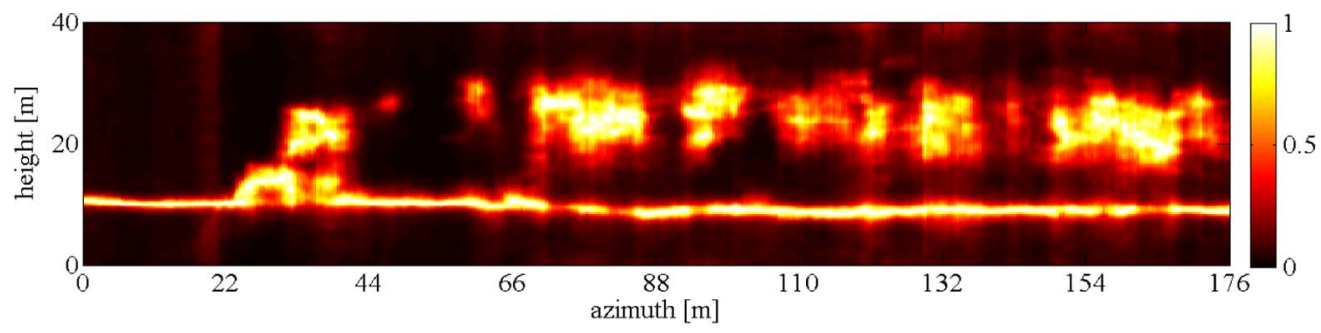

(a)

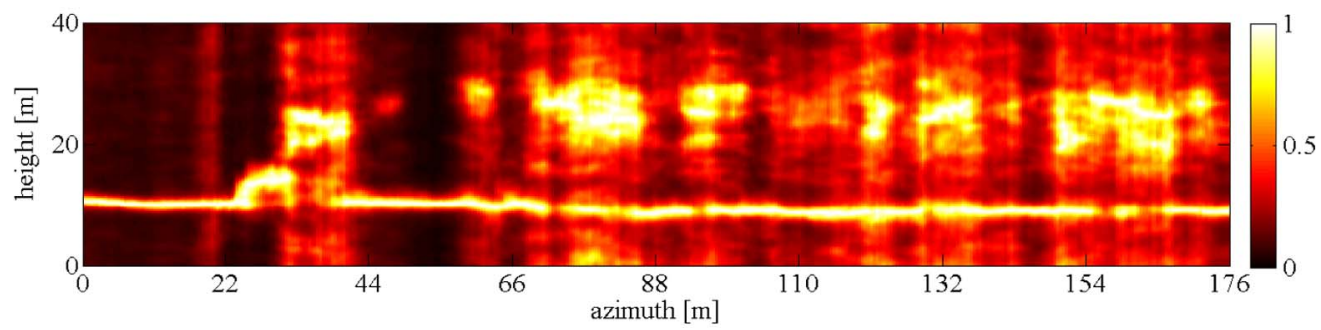

(b)

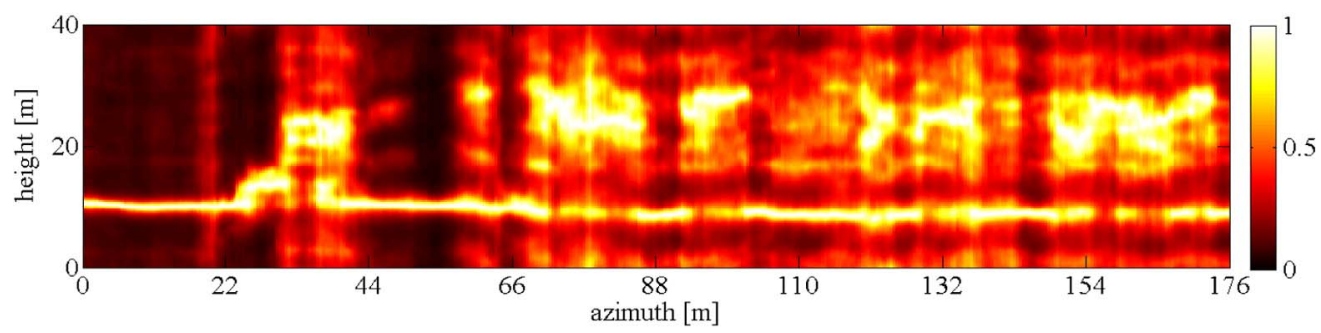

(c)

Fig. 12. Span of tomogram obtained by Capon's method as a function of azimuth and height (176 m by $40 \mathrm{~m}$ ) using a nine-by-nine window with (a) 21 , (b) 10 , and (c) 6 passes. Range distance: $4816.30 \mathrm{~m}$.

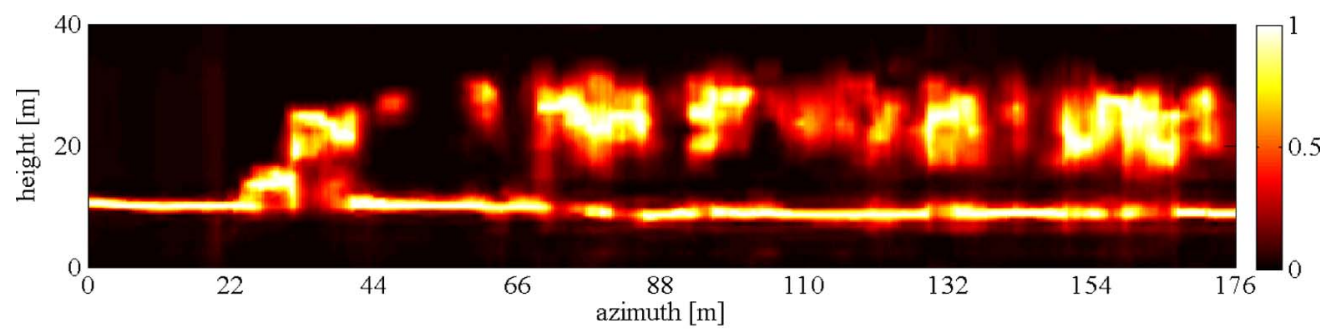

(a)

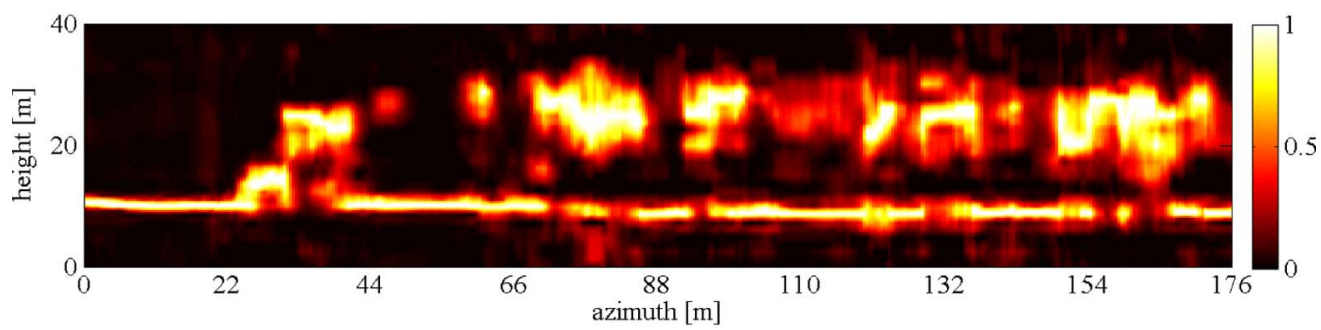

(b)

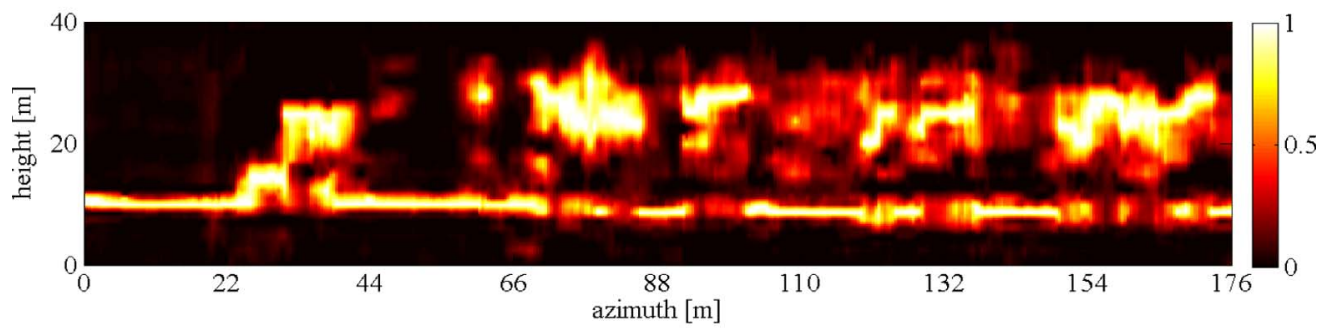

(c)

Fig. 13. Span of tomogram obtained by WCS as a function of azimuth and height (176 m by $40 \mathrm{~m}$ ) using a nine-by-nine window with (a) 21 , (b) 10 , and (c) 6 passes. Range distance: $4816.30 \mathrm{~m}$. 


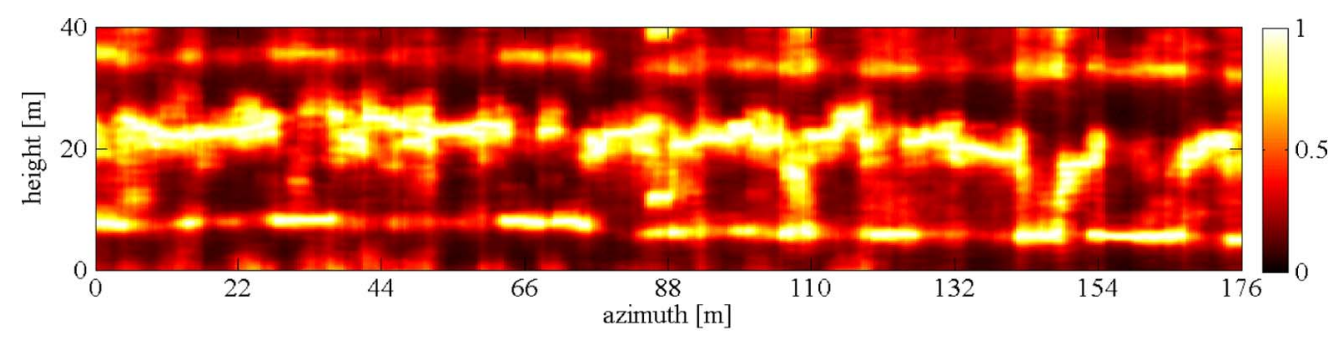

(a)

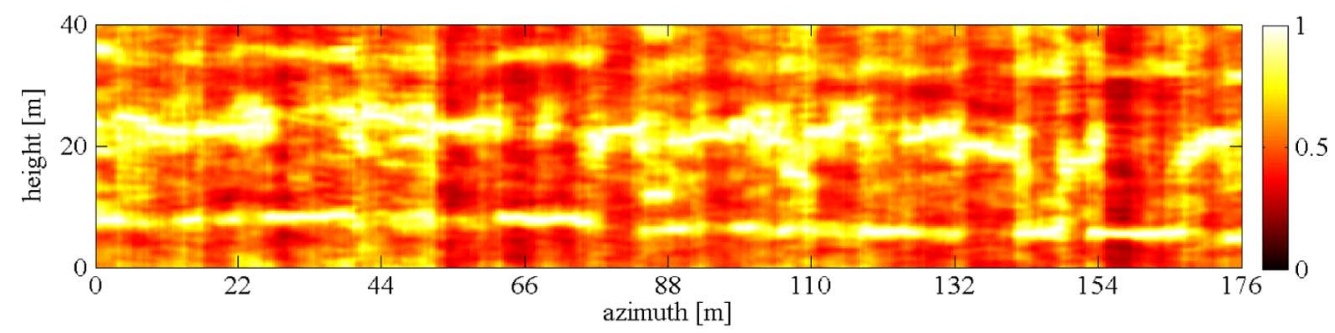

(b)

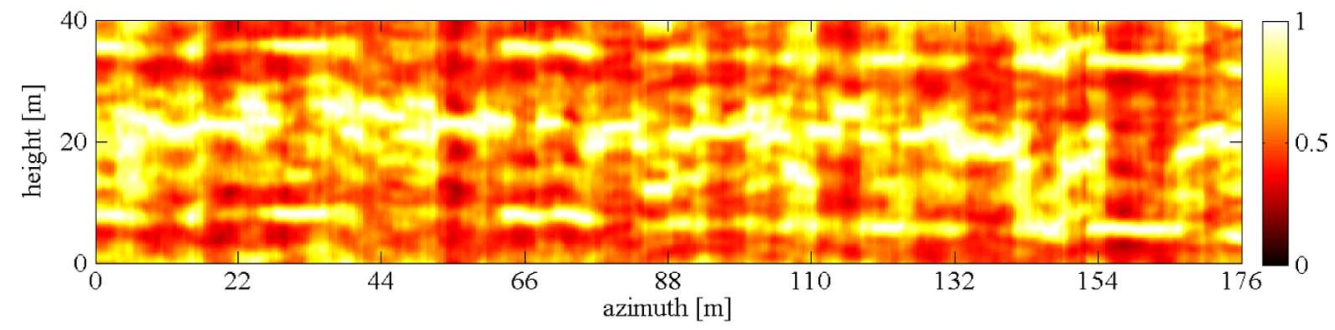

(c)

Fig. 14. Span of tomogram obtained by Fourier beamforming as a function of azimuth and height (176 $\mathrm{m}$ by $40 \mathrm{~m}$ ) using a nine-by-nine window with (a) 21 , (b) 10, and (c) 6 passes. Range distance: $4501.61 \mathrm{~m}$.

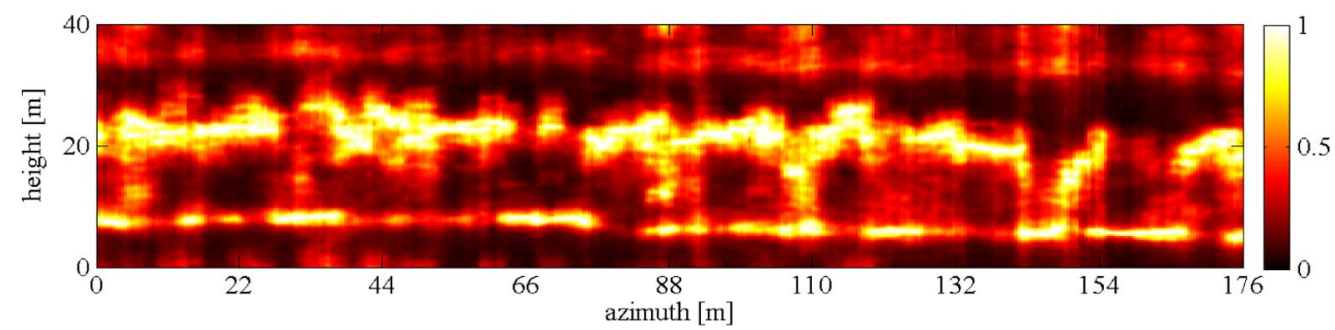

(a)

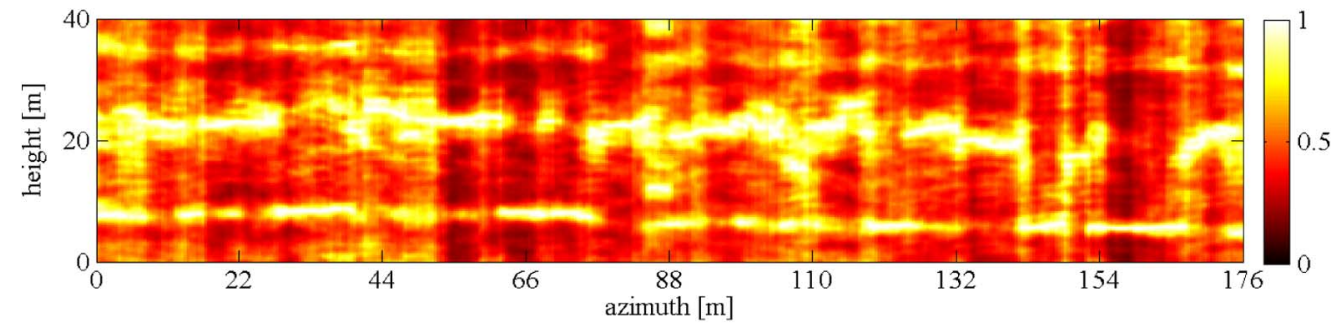

(b)

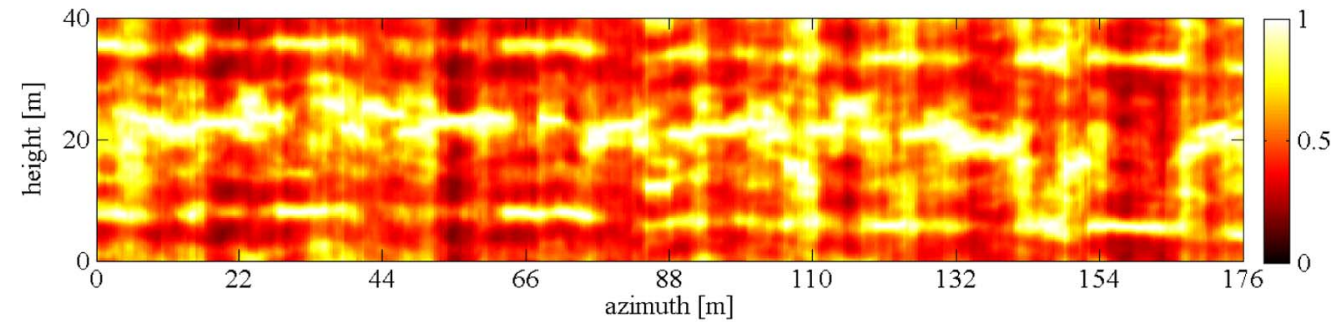

(c)

Fig. 15. Span of tomogram obtained by Capon's method as a function of azimuth and height (176 $\mathrm{m}$ by $40 \mathrm{~m}$ ) using a nine-by-nine window with (a) 21 , (b) 10 , and (c) 6 passes. Range distance: $4501.61 \mathrm{~m}$. 


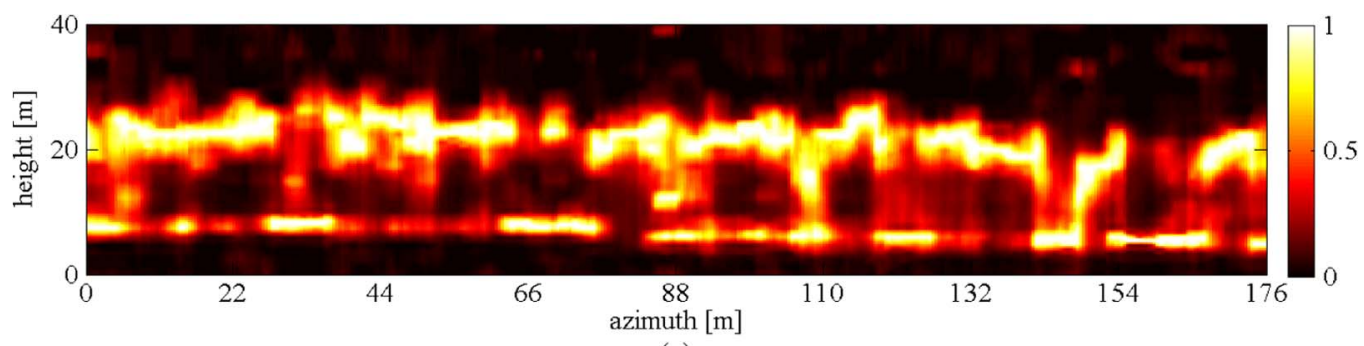

(a)

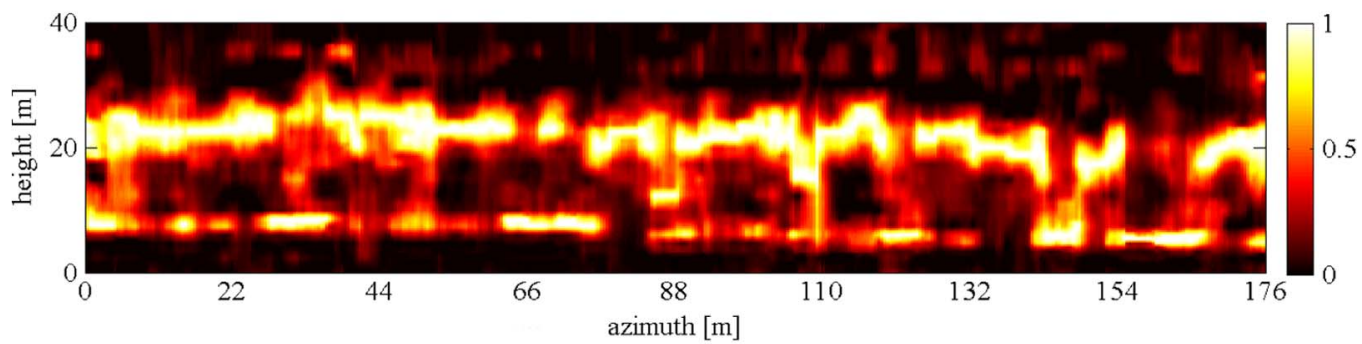

(b)

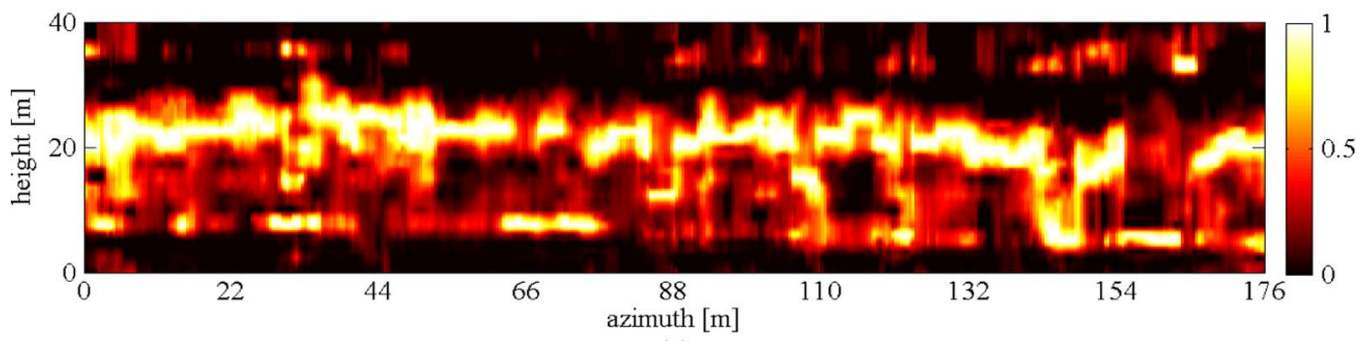

(c)

Fig. 16. Span of tomogram obtained by WCS as a function of azimuth and height (176 m by $40 \mathrm{~m}$ ) using a nine-by-nine window with (a) 21 , (b) 10 , and (c) 6 passes. Range distance: $4501.61 \mathrm{~m}$.

appropriately bounded, as the backscatter corresponding to the neglected observation space can be absorbed by $\varepsilon$ in $(7)$.

Finally, it is worth noting that, in the authors' experience, although the TV norm regularization promotes the removal of spurious spikes and aliasing-like artifacts, setting $\lambda_{2}=0$ also leads to satisfactory results.

\section{B. Experiments With Real Data}

For validation purposes, we selected 400 contiguous azimuth positions at two different range locations (indicated by the yellow rectangles and the red lines in Fig. 3). As a result, we obtained tomographic slices as a function of azimuth and height of dimensions $176 \mathrm{~m}$ by $40 \mathrm{~m}(n=128)$, respectively. In both cases, we took a nine-by-nine window. In Fig. 11, we used Fourier beamforming for a range distance of $4816.30 \mathrm{~m}$. Fig. 11(a)-(c) displays the normalized sum of the power distributions throughout polarimetric channels using the constellations $C 1-C 3$, respectively. Likewise, as presented in Fig. 12, we carried out the reconstruction with Capon's beamformer. Alternatively, Fig. 13 shows the results obtained via WCS using $\lambda_{1}=\lambda_{2}=0.5$ [see (16)], (3), and a Daubechies Symmlet wavelet with four vanishing moments and three levels of decomposition. Evidently, all methods bear comparison with each other for $C 1$ [see Figs. 11(a), 12(a), and 13(a)]. However, a reduction in the number of tracks, i.e., constellations $C 2-C 3$, enables us to reveal the robustness of the different methods. In contrast to WCS [Fig. 13(b) and (c)], these irregular baseline

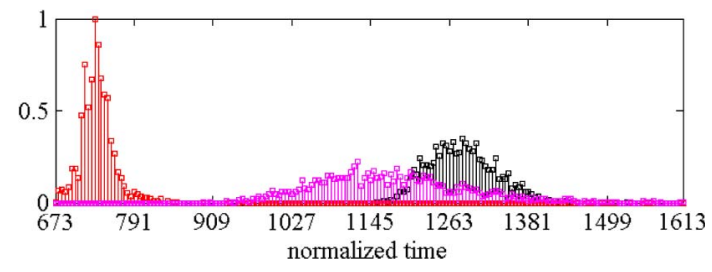

Fig. 17. Normalized histogram of the reconstruction time required for WCS (black: 21 passes; red: 10 passes; and magenta: 6 passes). The times have been normalized to the Fourier beamforming reconstruction time using 21 passes.

distributions cause Fourier beamforming [Fig. 11(b) and (c)] as well as Capon's method [Fig. 12(b) and (c)] to present more severe artifacts, just as observed with the simulated data. Figs. 14-16 show similar results at a nearer range $(4501.61 \mathrm{~m})$, so as to see the impact of the ambiguities even for $C 1$. Upon comparison, we observe that the WCS reconstruction exhibits the lowest ambiguity level.

\section{Computation Time}

Fig. 17 presents three histograms of the reconstruction time required for WCS. The different constellations $(C 1-C 3)$ were employed for simulating the corresponding multibaseline measurements at the far range. For every realization (2000 in total), one of the profiles from the left column of Fig. 2 was chosen uniformly at random. Again, the $\mathrm{SNR}=10 \mathrm{~dB}$, and the number of looks was 300 . The resulting times have been normalized to the Fourier beamforming reconstruction time using 21 passes. 
The solver that we used was CVX, which is a package for specifying and solving convex programs [50]. As conveyed by the histograms, besides incurring much more computation time, WCS is less predictable, due to the iterative nature of the algorithm. For large-scale processing, we refer the reader to [51].

\section{CONCLUSION}

In this paper, we have approached the 3-D reconstruction of forested areas from a CS perspective. We analyzed the effective cross-range power distribution in the wavelet domain and motivated the use of its corresponding bases. As a result, we achieved high resolution while attaining low ambiguity levels. Furthermore, we examined the actual sampling waveforms used for measuring the unknown power distribution. Thus, we pointed out the possibility of optimizing the acquisition geometry by means of a reduced set of irregular baselines. We have shown that, in contrast to traditional nonparametric spectral estimators, previous knowledge about the observation space proves beneficial for WCS. Also, as WCS inherently estimates the cross-range backscattered power simultaneously for all heights in the defined observation space, it is able to counter possible ambiguities. However, an erroneous range of heights can introduce undesired, yet well-bounded, artifacts. Lastly, we note that, even though research is currently being conducted in order to solve CS problems efficiently (see, for example, [51] and the references therein), the increase in computational complexity is significant.

\section{REFERENCES}

[1] A. Reigber and A. Moreira, "First demonstration of airborne SAR tomography using multibaseline L-band data," IEEE Trans. Geosci. Remote Sens., vol. 38, no. 5, pp. 2142-4152, Sep. 2000.

[2] S. Tebaldini and F. Rocca, "Multibaseline polarimetric SAR tomography of a boreal forest at P- and L-bands," IEEE Trans. Geosci. Remote Sens., vol. 50, no. 1, pp. 232-246, Jan. 2012.

[3] M. Nannini, R. Scheiber, R. Horn, and A. Moreira, "First 3-D reconstructions of targets hidden beneath foliage by means of polarimetric SAR tomography," IEEE Geosci. Remote Sens. Lett., vol. 9, no. 1, pp. 60-64, Jan. 2012.

[4] S. Tebaldini, "Algebraic synthesis of forest scenarios from multibaseline PolInSAR data," IEEE Trans. Geosci. Remote Sens., vol. 47, no. 12, pp. 4132-4142, Dec. 2009.

[5] F. M. O. Frey and E. Meier, "Tomographic imaging of a forested area by airborne multi-baseline P-band SAR," Sensors, vol. 8, no. 9, pp. 58845896, Sep. 2008

[6] O. Frey and E. Meier, "Analyzing tomographic SAR data of a forest with respect to frequency, polarization, and focusing technique," IEEE Trans. Geosci. Remote Sens., vol. 49, no. 10, pp. 3648-3659, Oct. 2011.

[7] M. M. d'Alessandro and S. Tebaldini, "Phenomenology of P-band scattering from a tropical forest through three-dimensional SAR tomography," IEEE Geosci. Remote Sens. Lett., vol. 9, no. 3, pp. 442-446, May 2012.

[8] J. Homer, I. Longstaff, and G. Callaghan, "High resolution 3-D SAR via multi-baseline interferometry," in Proc. IGARSS, May 1996, vol. 1, pp. 796-798.

[9] P. Pasquali, C. Prati, F. Rocca, M. Seymour, J. Fortuny, E. Ohlmer, and A. Sieber, "A 3-D SAR experiment with EMSL data," in Proc. IGARSS, Jul. 1995, vol. 1, pp. 784-786.

[10] F. Lombardini and A. Reigber, "Adaptive spectral estimation for multibaseline SAR tomography with airborne L-band data," in Proc. IGARSS, Jul. 2003, vol. 3, pp. 2014-2016.

[11] F. Lombardini, M. Pardini, G. Fornaro, F. Serafino, L. Verrazzani, and M. Costantini, "Linear and adaptive spaceborne three-dimensional SAR tomography: A comparison on real data," IET Radar, Sonar, Navigat., vol. 3, no. 4, pp. 424-436, Aug. 2009.

[12] S. Sauer, L. Ferro-Famil, A. Reigber, and E. Pottier, "Three-dimensional imaging and scattering mechanism estimation over urban scenes using dual-baseline polarimetric InSAR observations at L-band," IEEE Trans. Geosci. Remote Sens., vol. 49, no. 11, pp. 4616-4629, Nov. 2011.

[13] O. Frey and E. Meier, "3-D time-domain SAR imaging of a forest using airborne multibaseline data at L- and P-bands," IEEE Trans. Geosci. Remote Sens., vol. 49, no. 10, pp. 3660-3664, Oct. 2011.

[14] S. Guillaso and A. Reigber, "Polarimetric SAR tomography," in Proc. of POLINSAR, Frascati, Italy, Jan. 2005.

[15] M. Nannini, R. Scheiber, and A. Moreira, "Estimation of the minimum number of tracks for SAR tomography," IEEE Trans. Geosci. Remote Sens., vol. 47, no. 2, pp. 531-543, Feb. 2009.

[16] Y. Huang, L. Ferro-Famil, and A. Reigber, "Under-foliage object imaging using SAR tomography and polarimetric spectral estimators," IEEE Trans. Geosci. Remote Sens., vol. 50, no. 6, pp. 2213-2225, Jun. 2012.

[17] F. S. G. Fornaro and F. Soldovieri, "Three-dimensional focusing with multipass SAR data," IEEE Trans. Geosci. Remote Sens., vol. 41, no. 3, pp. 507-517, Mar. 2003.

[18] X. Zhu and R. Bamler, "Very high resolution spaceborne SAR tomography in urban environment," IEEE Trans. Geosci. Remote Sens., vol. 48, no. 12, pp. 4296-4308, Dec. 2010.

[19] F. Lombardini and M. Pardini, "3-D SAR tomography: The multibaseline sector interpolation approach," IEEE Geosci. Remote Sens. Lett., vol. 5, no. 4, pp. 630-634, Oct. 2008.

[20] S. Tebaldini, "Single and multipolarimetric SAR tomography of forested areas: A parametric approach," IEEE Trans. Geosci. Remote Sens., vol. 48, no. 5, pp. 2375-2387, May 2010.

[21] S. Cloude, "Polarization coherence tomography," Radio Sci., vol. 41, no. 4, pp. RS4017-1-RS4017-27, Aug. 2006.

[22] X. Zhu and R. Bamler, "Tomographic SAR inversion by L1-norm regularization-the compressive sensing approach," IEEE Trans. Geosci. Remote Sens., vol. 48, no. 10, pp. 3839-3846, Oct. 2010.

[23] A. Budillon, A. Evangelista, and G. Schirinzi, "Three-dimensional SAR focusing from multipass signals using compressive sampling," IEEE Trans. Geosci. Remote Sens., vol. 49, no. 1, pp. 488-499, Jan. 2011.

[24] X. Zhu and R. Bamler, "Super-resolution power and robustness of compressive sensing for spectral estimation with application to spaceborne tomographic SAR,” IEEE Trans. Geosci. Remote Sens., vol. 50, no. 1, pp. 247-258, Jan. 2012.

[25] E. Aguilera, M. Nannini, and A. Reigber, "Multisignal compressed sensing for polarimetric SAR tomography," IEEE Geosci. Remote Sens. Lett., vol. 9, no. 5, pp. 871-875, Sep. 2012.

[26] S. Samadi, M. Çetin, and M. Masnadi-Shirazi, "Multiple featureenhanced synthetic aperture radar imaging," in Proc. SPIE Defense Security Symp., 16th Algorithms Synthet. Aperture Radar Imagery, Apr. 2009, vol. 7337, pp. 73370I-1-73370I-10.

[27] L. Potter, E. Ertin, J. Parker, and M. Cetin, "Sparsity and compressed sensing in radar imaging," Proc. IEEE, vol. 98, no. 6, pp. 1006-1020, Jun. 2010.

[28] J. Wu, F. Liu, L. Jiao, and X. Wang, "Compressive sensing SAR image reconstruction based on Bayesian framework and evolutionary computation," IEEE Trans. Image Process., vol. 20, no. 7, pp. 1904-1911, Jul. 2011.

[29] S. Bhattacharya, T. Blumensath, B. Mulgrew, and M. Davies, "Synthetic aperture radar raw data encoding using compressed sensing," in Proc. IEEE RADAR Conf., May 2008, pp. 1-5.

[30] M. Lustig, D. Donoho, and J. Pauly, "Sparse MRI: The application of compressed sensing for rapid MR imaging," Magn. Reson. Med., vol. 58, no. 6, pp. 1182-1195, Dec. 2007.

[31] I. Loris, G. Nolet, I. Daubechies, and F. Dahlen, "Tomographic inversion using L1-norm regularization of wavelet coefficients," Geophys. J. Int., vol. 170, no. 1, pp. 359-370, Jul. 2007.

[32] R. Bamler and P. Hartl, "Synthetic aperture radar interferometry," Inverse Prob., vol. 14, no. 4, pp. R1-R54, Aug. 1998.

[33] E. Candès, "Compressive sampling," in Proc. Int. Congr. Math., Madrid, Spain, 2006, vol. 3, pp. 1433-1452.

[34] R. Baraniuk, "Compressive sensing," IEEE Signal Process. Mag., vol. 24, no. 4, pp. 118-121, Jul. 2007.

[35] D. Donoho, "Compressed sensing," IEEE Trans. Inf. Theory, vol. 52, no. 4, pp. 1289-1306, Apr. 2006.

[36] E. Candès, J. Romberg, and T. Tao, "Robust uncertainty principles: Exact signal reconstruction from highly incomplete frequency information," IEEE Trans. Inf. Theory, vol. 52, no. 2, pp. 489-509, Feb. 2006.

[37] E. Candès and T. Tao, "Near-optimal signal recovery from random projections: Universal encoding strategies?" IEEE Trans. Inf. Theory, vol. 52, no. 12, pp. 5406-5425, Dec. 2006.

[38] E. Candès and T. Tao, "Decoding by linear programming," IEEE Trans. Inf. Theory, vol. 51, no. 12, pp. 4203-4215, Dec. 2005. 
[39] E. Candès, J. Romberg, and T. Tao, "Stable signal recovery from incomplete and inaccurate measurements," Commun. Pure Appl. Math., vol. 59, no. 8, pp. 1207-1223, Aug. 2006.

[40] E. Candès, "The restricted isometry property and its implications for compressed sensing," Comptes Rendus Math., vol. 346, no. 9/10, pp. 589592, May 2008.

[41] E. Candès and J. Romberg, "Sparsity and incoherence in compressive sampling," Inverse Prob., vol. 23, no. 3, pp. 969-985, Jun. 2007.

[42] M. Rudelson and R. Vershynin, "On sparse reconstruction from Fourier and Gaussian measurements," Commun. Pure Appl. Math., vol. 61, no. 8, pp. 1025-1045, Aug. 2008.

[43] C. Sidney Burrus, R. Gopinath, and H. Guo, Introduction to Wavelets and Wavelet Transforms: A Primer. Upper Saddle River, NJ: Prentice-Hall, 1998.

[44] S. Mallat, A Wavelet Tour of Signal Processing. Burlington, MA: Academic, 2009.

[45] D. Linebarger, I. Sudborough, and I. Tollis, "Difference bases and sparse sensor arrays," IEEE Trans. Inf. Theory, vol. 39, no. 2, pp. 716-721, Mar. 1993.

[46] M. Nannini, A. Reigber, and R. Scheiber, "A study on irregular baseline constellations in SAR tomography," in Proc. EUSAR, Jun. 2010, pp. 1-4.

[47] J. Yang, Y. Zhang, and W. Yin, "A fast alternating direction method for TVL1-L2 signal reconstruction from partial Fourier data," IEEE J. Sel. Topics Signal Process., vol. 4, no. 2, pp. 288-297, Apr. 2010.

[48] R. Tibshirani, M. Saunders, S. Rosset, J. Zhu, and K. Knight, "Sparsity and smoothness via the fused lasso," J. R. Stat. Soc., B Stat. Methodol., vol. 67, no. 1, pp. 91-108, Feb. 2005.

[49] J. Hagberg, L. Ulander, and J. Askne, "Repeat-pass SAR interferometry over forested terrain," IEEE Trans. Geosci. Remote Sens., vol. 33, no. 2, pp. 331-340, Mar. 1995.

[50] M. Grant and S. Boyd, CVX: Matlab Software for Disciplined Convex Programming. [Online]. Available: http://cvxr.com/cvx

[51] S. Becker, E. Candès, and M. Grant, "Templates for convex cone problems with applications to sparse signal recovery," Math. Program. Comput., vol. 3 , no. 3, pp. 165-218, Aug. 2011

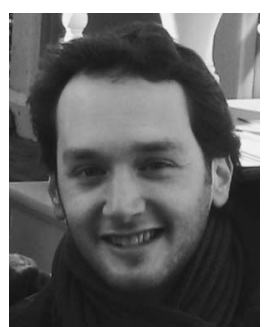

Esteban Aguilera was born in Mendoza, Argentina, on September 11, 1983. He received the Ingeniero degree in information systems engineering from the National Technological University, Mendoza, in 2007. He is currently working toward the Ph.D. degree in the Microwaves and Radar Institute (HR), German Aerospace Center (DLR), Wessling, Germany.

From 2008 to 2009, he was a Consultant to a wide range of information technology projects in Belgium and The Netherlands. His main research interests are in polarimetric synthetic aperture radar (SAR), SAR interferometry, and SAR tomography.

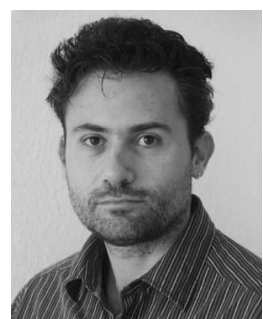

Matteo Nannini received the Laurea degree in telecommunication engineering from the University of Florence, Florence, Italy, in 2003, with a thesis done in collaboration with the Microwaves and Radar Institute (HR), German Aerospace Center (DLR), Wessling, Germany, and the Ph.D. degree from the University of Karlsruhe, Karlsruhe, Germany, in 2009, with a thesis on synthetic aperture radar (SAR) tomography.

Since 2004, he has been with HR, DLR. His main research activities are SAR processing, differential interferometry, tomography, and radar sounding.

Dr. Nannini was awarded, as coauthor, with the Best Paper Prize at the joint Systems Engineering for Autonomous Systems and Electro-Magnetic Remote Sensing Defence Technology Centres Technical Conference in Edinburgh, U.K., in 2009 and 2010, respectively.

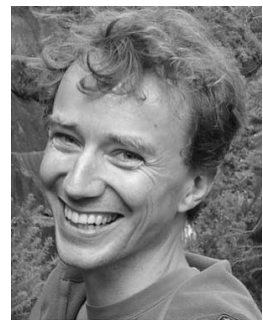

Andreas Reigber (M'02-SM'10) was born in Munich, Germany, in 1970. He received the Diploma degree in physics from the University of Constance, Konstanz, Germany, in 1997, the Ph.D. degree from the University of Stuttgart, Stuttgart, Germany, in 2001, and the Habilitation from Berlin University of Technology, Berlin, Germany, in 2008.

From 1996 to 2000, he was with the Microwaves and Radar Institute (HR), German Aerospace Center (DLR), Wessling, Germany, working in the field of polarimetric synthetic aperture radar (SAR) tomography. In 2001, he joined the Antenna, Radar and Telecom Laboratories, University of Rennes 1, Rennes, France, where he was a Postdoctoral Fellow on radar polarimetry and polarimetric interferometry. From 2002 to 2007, he was a Research Associate with the Computer Vision and Remote Sensing Laboratories, Berlin University of Technology. Since 2008, he has been with HR, DLR, where he is currently the Head of the SAR Technology Department and directing the airborne SAR activities of the institute. His current main research interests are the various aspects of multimodal SAR, like SAR interferometry, SAR polarimetry, SAR tomography, and time-frequency analyses, as well as filtering and classification aspects of high-resolution SAR data.

Dr. Reigber was the recipient of the European Conference on Synthetic Aperture Radar 2000 Student Prize Paper Award for an article on SAR remote sensing of forests, the IEEE TRANSACTIONS ON GEOSCIENCE AND Remote SENSING Prize Paper Award in 2001 for a work on polarimetric SAR tomography, and the IEEE Geoscience AND REMote SENSING LeTters Prize Paper Award in 2006 for a work on multipass SAR processing. 